\title{
Anisotropic high-field transverse differential mobility of holes in silicon
}

\author{
J. M. Hinckley a) and J. Singh \\ Department of Electrical Engineering and Computer Science, The University of Michigan, Ann Arbor, \\ Michigan 48109-2122
}

(Received 29 June 1995; accepted for publication 6 September 1995)

\begin{abstract}
Anisotropy of the silicon valence band does not lead to any significant anisotropy in the longitudinal hole transport properties, but we find that the transverse mobility is quite anisotropic. The transverse mobility represents the response of charge carriers to a small transverse electric field in the presence of a strong longitudinal field. A detailed, anisotropic Monte Carlo method has been applied to the calculation of the hole transverse differential mobility in silicon. The transverse differential mobility is studied both with regard to variations in the orientation, with respect to the crystalline axes, of the high longitudinal electric field, and with regard to variations in the transverse direction of the mobility, taken in the plane perpendicular to the high electric field. The anisotropy of the valence band causes the transverse differential mobility to strongly vary with respect to the electric field orientation. Symmetry considerations show that the transverse differential mobility is isotropic in the $\{100\}$ and $\{111\}$ planes and has twofold rotational symmetry in the $\{101\}$ planes. Our calculations bear this out. Furthermore, we show that the transverse mobility can be much different from the chordal mobility, in distinction to the case for isotropic band structures. (C) 1995 American Institute of Physics.
\end{abstract}

The characteristic anisotropy of semiconductor valence bands makes determination of the valence band structure in new semiconductors, inherently more complicated than making corresponding studies of the conduction band. Conventional experiments to determine the shape of the band structure, such as the cyclotron resonance experiment, usually involve some aspect of magnetotransport. These techniques have the drawback that as one lowers the sample temperature to reduce the amount of carrier scattering, the carrier density decreases sharply. In large band-gap semiconductors, this can cause virtually complete carrier freezeout, rendering the method unusable.

As an alternative to magnetotransport methods, we have examined various aspects of room-temperature, high electric field, zero magnetic field hole transport, looking for phenomena in which the anisotropy of the valence band is clearly manifested. In addition to avoiding carrier freezeout in large band-gap materials, by working at room temperature, such an approach would be attractive by virtue of its not requiring the generation of high magnetic fields.

Most familiar high-field hole transport characteristics, such as the drift velocity, are only slightly affected by the valence band anisotropy. ${ }^{1}$ This is due to the fact that during drift, a typical hole scatters over a large portion of phase space, thus sampling the band structure in virtually all directions. Thus, drift velocity experiments are not generally useful in determining valence band structure details. However, in the present work, we have found, on the basis of Monte Carlo calculations, that the high-field transverse differential mobility of holes, in silicon, does have a pronounced anisotropy with respect to the electric field orientation. Thus transverse mobility represents the response of charge carriers to a small transverse electric field in the presence of a strong longitudinal field, and is the ratio of the small velocity com-

${ }^{a)}$ Electronic mail: ncko@engin.umich.edu ponent, in the direction of the transverse field, and the transverse field magnitude.

In addition to its potential application in investigating valence band structures, the transverse differential mobility is pertinent to both the analysis of transverse microwave conductivity in semiconductors ${ }^{2}$ and the determination of the transverse diffusion noise characteristics. ${ }^{3}$ For isotropic band structures, the transverse differential mobility equals the longitudinal chordal mobility, but as pointed out in Ref. 3, this is generally not true in anisotropic band structures. At very low fields, in cubic materials (with anisotropic band structures), the transverse differential mobility, the longitudinal differential mobility and the longitudinal chordal mobility are all equal to the Ohmic mobility. The rough equality of the transverse differential mobility and the longitudinal chordal mobility has been used for analysis of the diffusion noise of warm holes, in $p$-Ge for fields up to $1 \mathrm{kV} / \mathrm{cm}$ at $T=300 \mathrm{~K}$. However, this equality certainly is not true at substantially higher fields, where the nature of transverse differential mobility is largely unknown experimentally. ${ }^{3}$ Therefore, aside from band structure investigations, the general findings presented in this letter are of relevance to transverse microwave conductivity and transverse diffusion noise, for longitudinal electric fields on the order of $50 \mathrm{kV} / \mathrm{cm}$ in magnitude.

We have applied our Monte Carlo method ${ }^{5-7}$ to the calculation of the transverse differential mobility of holes in silicon for different directions of high electric field, at a field magnitude of $50 \mathrm{kV} / \mathrm{cm}$ and temperature of $300 \mathrm{~K}$. The method accounts for the valence band anisotropy by using a $6 \times 6 k \cdot p$ Hamiltonian, with spin-orbit coupling. This gives both the anisotropic band structure of the heavy, light, and splitoff bands, and the eigenvectors, which in turn, are used to calculate scattering rates that are, themselves, anisotropic. The scattering rates are calculated for both intraband and interband scattering, involving all three hole bands. The scattering mechanisms used are acoustic phonon and nonpolar 


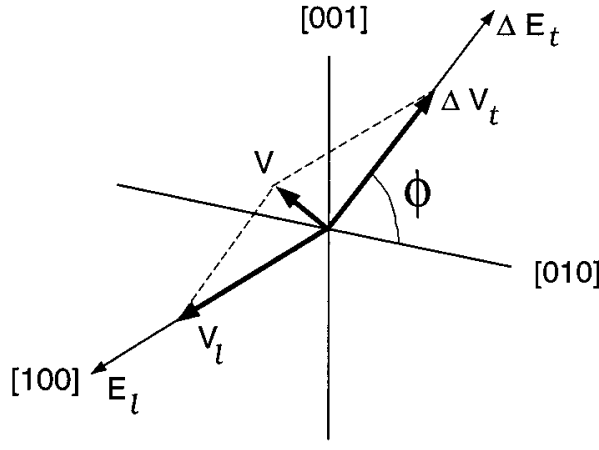

FIG. 1. Schematic geometry of longitudinal and transverse electric field components and the resulting carrier velocity components. For this example, $\mathbf{E}_{/} \|[100]$. The longitudinal and transverse directions are defined by the orientations of $\mathbf{E}_{/}$and $\Delta \mathbf{E}_{t}$, respectively. The transverse component $\Delta \mathbf{E}_{t}$, lies in the (100) plane, perpendicular to $\mathbf{E}_{/}$, at an angle $\phi$ with respect to the $y$-axis. The projections of the drift velocity $v$, on the longitudinal and transverse directions are $v_{l}$ and $\Delta v_{t}$, respectively. The magnitude of $\Delta \mathbf{E}_{t}$, relative to that of $\mathbf{E}_{/}$, is exaggerated in this figure for clarity. For the calculations of $\mu_{t}=v_{\ell} / \Delta E_{t}$, the values of $E_{\ell}$ and $\Delta E_{t}$ used were 50 and 2 $\mathrm{kV} / \mathrm{cm}$, respectively.

optical phonon scattering. Thus, we are considering hole transport in pure silicon only.

For a given orientation of a large electric field $\mathbf{E}_{/}$, a relatively small electric field vector $\Delta \mathbf{E}_{t}$ is added, in some direction lying in the plane perpendicular to $\mathbf{E}_{\ell}$. The geometry involved is shown schematically in Fig. 1, for the case of $\mathbf{E}_{/} \|[100]$. The orientation of $\mathbf{E}_{/}$defines the longitudinal direction " $\ell$ " and the orientation of $\Delta \mathbf{E}_{t}$ defines the transverse direction ' $t$.' ' In this work, we investigate the three cases: $\mathbf{E}_{/}\left\|[100], \mathbf{E}_{/}\right\|[111]$, and $\mathbf{E}_{/} \|[101]$. The Monte Carlo calculates the drift velocity vector $v$, corresponding to the compound electric field. The drift velocity $v$, has projections $v_{\ell}$ and $\Delta v_{t}$, on the longitudinal and transverse directions, respectively. The transverse differential mobility $\mu_{t}$ is calculated as the ratio of $\Delta v_{t}-\Delta E_{t}$. The value of $\mu_{t}$ will depend upon the orientation of $\mathbf{E}_{\ell}$. In the cases of $\mathbf{E}_{\ell} \|[100]$ and $\mathbf{E}_{/} \|[111]$, the respective values of $\mu_{t}, \mu_{t}^{[100]}$, and $\mu_{t}^{[111]}$, are independent of the orientation (i.e., the angle $\phi$ in Fig. 1) of $\Delta \mathbf{E}_{t}$, in the plane perpendicular to $\mathbf{E}_{\ell}$. This can be demonstrated on the basis of symmetry, and was directly confirmed by us in a series of simulations. However, for $\mathbf{E}_{\ell} \|[101], \mu_{t}$ will vary with the orientation of $\Delta \mathbf{E}_{t}$. For $\Delta \mathbf{E}_{t} \|[010], \mu_{t}=\mu_{t 1}^{[101]}$ and for $\Delta \mathbf{E}_{t} \|\left[\overline{101]}, \quad \mu_{t}=\mu_{t 2}^{[101]} ; \mu_{t 1}^{[101]} \neq \mu_{t 2}^{[101]}\right.$. For intermediate orientations of $\Delta \mathbf{E}_{t}, \mu_{t}^{[101]}=\cos ^{2} \phi \mu_{t 1}^{[101]}+\sin ^{2} \phi \mu_{t 2}^{[101]}$, where $\phi$ is the angle between the $y$-axis and any chosen arbitration direction perpendicular to [101]. This result, too, is based on symmetry of the crystal and was well-confirmed in our simulations.

Figure 2 shows the results of our calculations of the transverse velocity of holes in silicon at room temperature. For these calculations, $E_{\ell}=50 \mathrm{kV} / \mathrm{cm}$ was used. For each value of magnitude and of orientation $\Delta \mathbf{E}_{t}$, a calculation involved the simulation of one particle over a $100 \mathrm{~ns}$ time of flight.

From these data, the following transverse differential mobilities are obtained

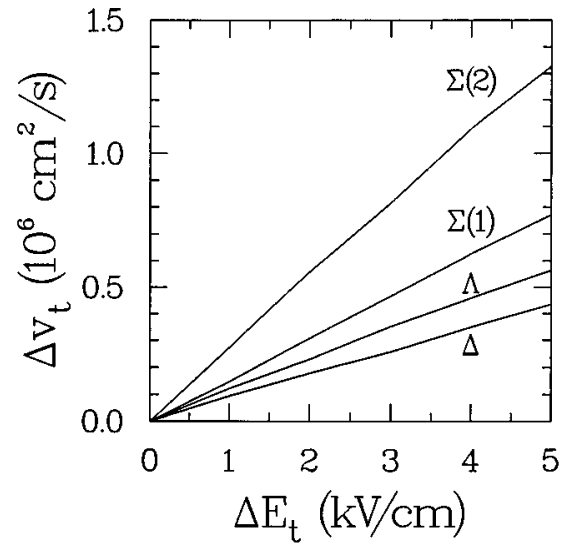

FIG. 2. Transverse differential mobility of holes in silicon. For this set of data, the longitudinal field $E_{\ell}$, is $50 \mathrm{kV} / \mathrm{cm}$ and $T=300 \mathrm{~K}$. Labeling $\Sigma, \Lambda$, $\Delta$ refers to the orientation of $E_{\ell}: \Sigma: \mathbf{E}_{/}\left\|[101] ; \Lambda: \mathbf{E}_{/}\right\|[111] ; \Delta: \mathbf{E}_{/} \|[100]$. For the [101] field orientation, the two nonequivalent transverse directions are $\Delta \mathbf{E}_{t} \|[010]$, labeled $\Sigma(1)$, and $\Delta \mathbf{E}_{t} \|[\overline{1} 01]$, labeled $\Sigma(2)$. Values of transverse mobility reported in this letter are obtained from these data as the ratio of $\Delta v_{t}$ to $\Delta E_{t}$ at $\Delta E_{t}=2 \mathrm{kV} / \mathrm{cm}$.

$$
\begin{array}{ll}
\mu_{t}^{[100]}=86 \quad \mathrm{~cm}^{2} / \mathrm{V} \mathrm{s}, \\
\mu_{t}^{[111]}=113 \quad \mathrm{~cm}^{2} / \mathrm{V} \mathrm{s}, \\
\mu_{t 1}^{[101]}=171 \quad \mathrm{~cm}^{2} / \mathrm{V} \mathrm{s}, \\
\mu_{t 2}^{[101]}=279 \quad \mathrm{~cm}^{2} / \mathrm{V} \mathrm{s} .
\end{array}
$$

These differential mobilities are calculated from the data in Fig. 2 by taking the ratio $\Delta v_{t} / \Delta E_{t}$ at $\Delta E_{t}=2 \mathrm{kV} / \mathrm{cm}$, where the $\Delta v_{t}$ vs $\Delta E_{t}$ relations for all curves are linear. For comparison with the longitudinal chordal mobility, $\mu_{c}$ $=v / / E_{\ell}$, such as one could validly use in an isotropic material (or at much lower fields), the corresponding chordal mobilities from our Monte Carlo calculations at $E_{\ell}=50$ $\mathrm{kV} / \mathrm{cm}$ are

$$
\begin{aligned}
& \mu_{c}^{[100]}=160 \quad \mathrm{~cm}^{2} / \mathrm{V} \mathrm{s}, \\
& \mu_{c}^{[111]}=134 \quad \mathrm{~cm}^{2} / \mathrm{V} \mathrm{s}, \\
& \mu_{c}^{[101]}=127 \quad \mathrm{~cm}^{2} / \mathrm{V} \mathrm{s} .
\end{aligned}
$$

It can be seen that the longitudinal chordal mobilities and the proper transverse differential mobilities bear no relationship to one another, under high field conditions.

At high fields, the behavior of the chordal mobility is primarily determined by the onset of drift velocity saturation. On the other hand, the transverse differential mobility is determined, in large part, by the reciprocal conductivity effective mass in the transverse direction. In order to study this connection, we have calculated average reciprocal conductivity effective mass tensors through Monte Carlo simulations. For a given electric field $\mathbf{E}_{\lambda}\left(\Delta \mathbf{E}_{t}=0\right.$ for these calculations) in one of the three orientations $[h k l]=[100],[111],[101]$, the six distinct averaged tensor elements obtained are 


$$
\left\langle\frac{m_{0}}{m^{*}}\right\rangle_{i j}^{[h k l]}=\frac{m_{0}}{\hbar^{2}}\left\langle\frac{\partial}{\partial k_{i}} \frac{\partial}{\partial k_{j}} \mathscr{C}_{n}(\mathbf{k})\right\rangle_{n, \mathbf{k}}, \quad i, j=x, y, z,
$$

where $\mathscr{E}$ is the carrier energy. The Monte Carlo performs the averaging over the bands, $n$, and the wave vector $\mathbf{k}$, with respect to the hole distribution function. For a given transverse direction $t$, with unit vector $\hat{e}=e_{1} \hat{x}+e_{2} \hat{y}+e_{3} \hat{z}$, the reciprocal mass is calculated by the double product

$$
\left\langle\frac{m_{0}}{m^{*}}\right\rangle_{t}^{[h k l]}=\sum_{i, j} e_{i}\left(\frac{m_{0}}{m^{*}}\right)_{i j}^{[h k l]} e_{j} .
$$

The calculated average transverse reciprocal masses are:

$$
\begin{aligned}
& \left\langle\frac{m_{0}}{m^{*}}\right\rangle_{t}^{[100]}=2.50, \\
& \left\langle\left.\frac{m_{0}}{m^{*}}\right|_{t} ^{[111]}=2.77,\right. \\
& \left\langle\left.\frac{m_{0}}{m^{*}}\right|_{t 1} ^{[101]}=2.56,\right. \\
& \left\langle\left.\frac{m_{0}}{m^{*}}\right|_{t 2} ^{[101]}=3.27 .\right.
\end{aligned}
$$

The dependence of the averaged reciprocal conductivity effective mass on field orientation is qualitatively similar to that of the transverse differential mobility. This helps to explain why $\mu_{t 1}^{[101]}<\mu_{t 2}^{[101]}$, which is contrary to what might otherwise be expected. Considering the heavy hole band, from the center of the Brillouin zone, the $[010](t 1)$ direction has a lower conductivity effective mass than does the [101] $(t 2)$ direction. This would lead one to expect that $\mu_{t 1}^{[101]}>\mu_{t 2}^{[101]}$, contrary to our findings. However, the highfield hole distribution averaged masses given above, show a higher mass in the $[010](t 1)$ direction than in the $[101](t 2)$ direction. This is a consequence of the displacement of the hot carrier distribution from the Brillouin zone center, which thus samples, on average, a different portion of the band structure. This is clarified in Fig. 3, which shows two cross sections through the heavy hole constant energy surface, perpendicular to [101], the direction of $\mathbf{E}_{\ell}$. The energy of these contours is $69 \mathrm{meV}$, which from our simulations, is the average heavy hole energy for $\mathbf{E}_{/}=50 \mathrm{kV} / \mathrm{cm}$. The dashed curve is the familiar cross section through the center of the constant energy surface, showing higher mass in the $[\overline{101}](t 2)$ direction than in the $[010](t 1)$ direction. The solid curve is a cross section, in a plane parallel to the first, offset from the origin by a vector $k=0.05$ $\AA^{-1}(1 / \sqrt{2}, 0,1 / \sqrt{2})$. This is more representative of the portion of the band structure that primarily determines the transverse characteristics, and shows a higher mass in the $[010](t 1)$ direction than in the $[\overline{101}](t 2)$ direction.

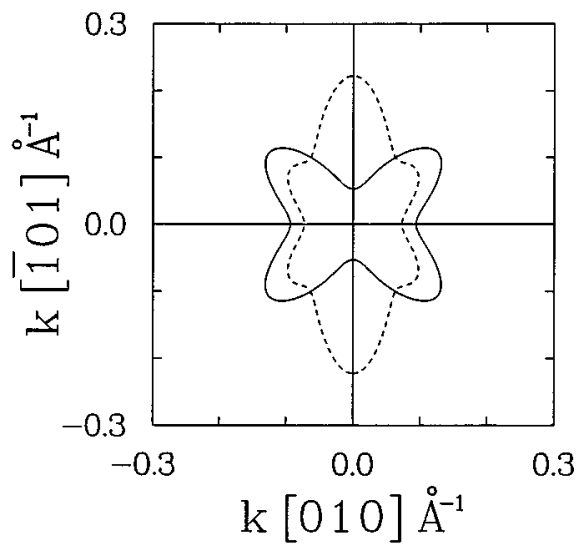

FIG. 3. Cross sections through the $69 \mathrm{meV}$ heavy hole constant energy surface. The dotted curve is the familiar cross section in the (101) plane, passing through the origin of the Brillouin zone. The solid curve is also in a (101) plane, but is displaced from the origin by a wave vector $k$ $=0.05 \AA^{-1}(1 / \sqrt{2}, 0,1 / \sqrt{2})$. The conductivity effective mass in this plane is a strong function of the displacement of the plane from the origin.

In summary, while at low fields, the transverse differential mobility is reasonably approximated by the longitudinal chordal mobility, at high fields, these quantities are quite different. We have presented a method of calculating the transverse differential mobility, incorporating the full nature of the anisotropies of the valence band structure and the scattering rates. The results are different from expectations based on the band structure about $k=0$, being strongly dependent on the displacement of the hot carrier distribution away from $k=0$.

Silicon was studied in this work, because its properties and material parameters are very well established, thereby lending high quantitative credibility to the new effects that we have presented. Due to the qualitative similarity of the crystal structure of silicon, germanium, diamond, and various III-V semiconductors, we expect that qualitatively similar results for the transverse differential mobility would be found in these other materials, as well. This work points out the need for correct accounting of the transverse differential mobility in the analysis of high-field transverse microwave conductivity and diffusion noise, as well as suggesting a means of probing the band structure of new high band-gap semiconductors.

This work was supported by the U. S. Air Force (Grant No. AFOSR-91-0349) and by the U. S. Army URI Program (DAAL03-92-G-0109).

${ }^{1}$ C. Jacoboni and L. Reggiani, Rev. Mod. Phys. 55, 645 (1983).

${ }^{2}$ Y. K. Pozhela, Hot-Electron Transport in Semiconductors, edited by L. Regiani (Springer, Berlin, 1984), Chap. 4.

${ }^{3}$ J.-P. Nougier, Physica 64, 209 (1973).

${ }^{4}$ J.-P. Nougier and M. Rolland, Phys. Rev. B 8, 5728 (1973).

${ }^{5}$ J. M. Hinckley and J. Singh, Phys. Rev. B 41, 2912 (1990).

${ }^{6}$ J. M. Hinckley, Ph.D. thesis, University of Michigan, Ann Arbor, 1990

${ }^{7}$ J. M. Hinckley and J. Singh, J. Appl. Phys. 76, 4192 (1994). 